\title{
Approximate sequential Bayesian filtering to estimate Rn-222 emanation from Ra-226 sources from spectra
}

\author{
Florian Mertes ${ }^{1}$, Stefan Röttger ${ }^{1}$, Annette Röttger ${ }^{1}$ \\ ${ }^{1}$ Physikalisch-Technische Bundesanstalt, Bundesallee 100, Braunschweig, Germany \\ florian.mertes@ptb.de
}

\begin{abstract}
Summary:
A new approach to assess the emanation of ${ }^{222} \mathrm{Rn}$ from ${ }^{226} \mathrm{Ra}$ sources based on measurements of the residual ${ }^{222} \mathrm{Rn}$ is presented. The method incorporates the dynamics into the inference procedure, rather than resorting to previously available steady-state approximations. The algorithm is based on approximate Bayesian filtering in a switched linear dynamical system to identify regimes of changing emanation behavior from a time-series of spectral data.
\end{abstract}

Keywords: State-Space model, switched linear dynamical system, Rn-222, integrating measurements

\section{Introduction}

For the calibration of ${ }^{222} \mathrm{Rn}$ measurement devices at low activity concentrations, decaying ${ }^{222} \mathrm{Rn}$ reference atmospheres produced through gaseous standards do not yield satisfactory statistical uncertainties. A different approach to realize reference atmospheres of low activity concentrations is provided by ${ }^{222} \mathrm{Rn}$ emanation sources. Emanation sources are ${ }^{226}$ Ra sources constructed so that some fraction of the generated ${ }^{222} \mathrm{Rn}$ is released from them. In [1, 2], an approach to measure the released amount of ${ }^{222} \mathrm{Rn}$ based on measuring the residual ${ }^{222} \mathrm{Rn}$ in the source is presented. However, this approach is only valid for times in which steady state has been reached. Moreover, it has been suggested that environmental conditions can impact the emanation behavior, which leads to erroneous results when assuming steady state. In the following, a new approach is presented based on Gaussian sum filtering in a switched linear dynamical system (SLDS) which more accurately models the emanation behavior, given the deterministic dynamics of the radioactive decay, with the possibility for on-line operation. Additionally, the method allows one to probabilistically identify regimes of constant emanation behavior.

\section{Model and filtering algorithm}

The basis of the new method is to model the ${ }^{226} \mathrm{Ra}$ source as a switched linear dynamical system, in which its latent state vector $x \in \mathbb{R}^{n \times 1}$, $x=\left[A_{R n-222}^{S}, A_{R a-226}^{S}, \eta, \frac{d \eta}{d t}\right]^{T}$, where $A_{i}^{S}$ is the activity of the $i$-th nuclide in the source and $\eta$ is the number of escaping ${ }^{222} \mathrm{Rn}$ atoms per unit time, evolves through the Itō stochastic differential equations (1).

$$
d x=F_{s} x d t+L_{s} d W_{s, t}
$$

where $F_{s} \in \mathbb{R}^{n \times n}$ is the fundamental matrix of the $s$-th model and $L_{s} \in \mathbb{R}^{n \times 1}$ is a matrix which controls how the increments of a Wiener-process $d W_{s, t}$ of power-spectral density $Q_{s} \in \mathbb{R}$ enter the system. The index $s$ of the active dynamics is modeled as a discrete Markov process with transition matrix $\Pi$. For each $s \in \mathbb{N}$, the solution to (1) is an initial value problem [3] and resembles a Gaussian process for Gaussian $x_{0} . F_{s}=F$ and $L_{S}=L$ are chosen to be

$F=\left[\begin{array}{cccc}-\lambda_{R n-222} & \lambda_{R n-222} & -\lambda_{R n-222} & 0 \\ 0 & -\lambda_{R a-226} & 0 & 0 \\ 0 & 0 & 0 & 1 \\ 0 & 0 & 0 & -\gamma\end{array}\right], L=\left[\begin{array}{l}0 \\ 0 \\ 0 \\ 1\end{array}\right]$

Generally, measurements with spectrometric devices $y \in \mathbb{R}^{m \times 1}$ (2) are performed over non-overlapping intervals $l_{k} \in \mathbb{R}$ indexed by $k \in \mathbb{N}$, such that $t_{k} \in T$, where $T$ represents the set of measurement time instants.

$$
y_{k, l_{k}}=H \int_{0}^{l_{k}} x\left(t_{k}+\tau\right) d \tau+r_{k}
$$

where $H \in \mathbb{R}^{m \times n}$ maps the state integral to the measurement space, $r$ is an uncorrelated Gaussian white noise sequence of variance $R_{k}$ indexed by $k$ that is computed from the observed $y_{k}$ (Gaussian approximation to counting statistics). This approximation is crucial, since it preserves the conjugacy of the model. $H$ is assumed to be deterministic. Given a time-series of spectra, we are interested in the filtering distribution $p\left(x_{k}, s_{k} \mid y_{0: k}, H\right)=p\left(x_{k} \mid s_{k}, y_{0: k}, H\right) p\left(s_{k} \mid y_{0: k}, H\right)$, which is defined recursively $[3,4]$, since both $x$ and $s$ are Markov processes. $y$ is a linear transformation of $x$, so for given $s_{k}, x_{k}$ and $y_{k}$ are jointly Gaussian. 


$$
\begin{aligned}
& p\left(x_{k}, y_{k} \mid s_{k}, H\right)= \\
& \mathcal{N}\left(\left[\begin{array}{c}
\mu_{x_{k}} \\
K_{l_{k}} \mu_{x_{k}}
\end{array}\right],\left[\begin{array}{cc}
\Sigma_{x_{k}} & \Sigma_{x_{k}} K_{l_{k}}^{T} \\
K_{l_{k}} \Sigma_{x_{k}} & K_{l_{k}} \Sigma_{x_{k}} K_{l_{k}}^{T}+J_{s_{k}, l_{k}}+R_{k}
\end{array}\right]\right)
\end{aligned}
$$

with

$K_{l_{k}}=H \int_{0}^{l_{k}} e^{F \tau} d \tau, J_{s_{k}, l_{k}}=\int_{0}^{l_{k}} K_{l_{k}-\tau} L Q_{s_{k}} L^{T} K_{l_{k}-\tau}^{T} d \tau$.

For the specific $F, L$ and $Q$ in the models, the discretization of (1), $K_{l_{k}}$ and $J_{s_{k}, l_{k}}$ are available analytically and were implemented using symbolic computation, using the diagonalizability of $F$. To obtain the approximate filtering distributions, Algorithm 1 in [4] was adapted to include the additional terms $K_{l_{k}}$ and $J_{s_{k}, l_{k}}$. As discussed in [4], it is infeasible to compute the exact filtering distribution. In our implementation, the arising Gaussian mixtures are collapsed to smaller mixtures based on an upper bound of the KL-divergence [5], where we chose to collapse to 3 Gaussian components per model. The unknown parameters $(Q, \Pi, \gamma)$ are tuned with respect to approximate maximum marginal likelihood [3].

\section{Experimental Results}

The SLDS approach was chosen because the time-series of interest is comprised of regimes of constant $\eta$ and those of changing $\eta$, where it is of interest to know when stable regimes are reached. The choice was made to model this behavior by having two linear dynamic models, one of them with fully deterministic dynamics e.g. $Q_{1}=0$.

For the collection of data, an electroplated ${ }^{226} \mathrm{Ra}$ (104.4 \pm 0.4$) \mathrm{Bq}$ source was mounted on top of a high-purity Germanium $\gamma$-ray detector, inside of a climate chamber. Spectra were recorded over the course of approx. 85 days in intervals of $10800 \mathrm{~s}$ live time. At specific times, the relative humidity was changed to induce changes in $\eta$. From each $\gamma$-ray spectrum, the total number of counts that were recorded at energies over $200 \mathrm{keV}$ was calculated, a background count rate was subtracted, and the algorithm was applied to the result. The threshold of $200 \mathrm{keV}$ was chosen because above this threshold the spectrum is only made up of events that are due to the background or the short-lived ${ }^{222} \mathrm{Rn}$ progeny (SLP) within the source. The SLP is assumed to always be in equilibrium with ${ }^{222} \mathrm{Rn}$, which is a valid approximation on these time-scales.

Figure 1 shows the raw-counts that were computed from the spectra (input data), the relative humidity inside the chamber as measured by a SHT-35 sensor (red curve), the inferred filtering distributions and the results that would have been obtained from the method in $[1,2]$. It can be observed from these results that once the dynamics of ingrowth are modeled in this way it becomes apparent that the methods in $[1,2]$ lead to deviations from the true value. The new method extends the validity to regimes of nonconstant $\eta$ and allows for an estimate of the naturally expected increased uncertainty in these regimes. Moreover, all obvious switching points within the time-series are detected.
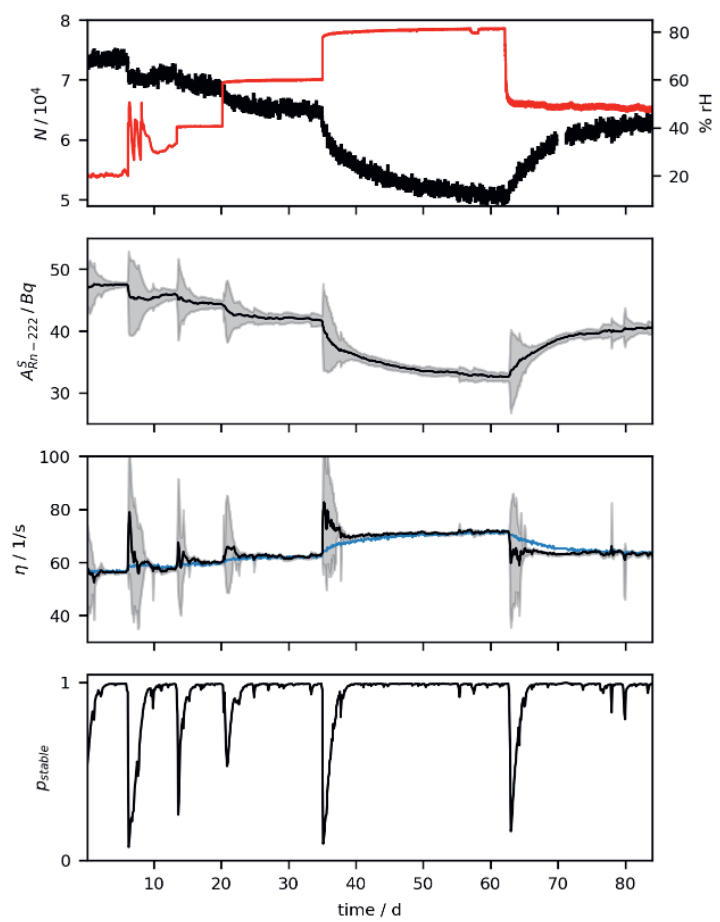

Figure 1: Filter output (black) with estimated $90 \%$ quantiles (grey) and comparison with results of the methods $[1,2]$ on the same dataset (blue)

\section{Acknowledgment}

This project 19ENV01 traceRadon has received funding from the EMPIR programme co-financed by the Participating States and from the European Union's Horizon 2020 research and innovation programme.

\section{References}

[1] D. Linzmaier, A. Röttger, Development of a lowlevel radon reference atmosphere, Applied Radiation and Isotopes, 81, 208-211, (2013), doi: 10.1016/j.apradiso.2013.03.032

[2] F. Mertes, S. Röttger, A. Röttger, A new primary emanation standard for Radon-222, Applied Radiation and Isotopes, 156, (2020), doi: 10.1016/j.apradiso.2019.108928

[3] S. Särkkä, A. Solin, Applied Stochastic Differential Equations, Cambridge University Press, (2019)

[4] D. Barber, Expectation Correction for Smoothed Inference in Switched Linear Dynamical Systems, Journal of Machine Learning Research, (2006)

[5] A. R. Runalls, Kullback-Leibler Approach to Gaussian Mixture Reduction, IEEE Transactions on Aerospace and Electronic Systems, 43, (2007), doi: 10.1109/TAES.2007.4383588 